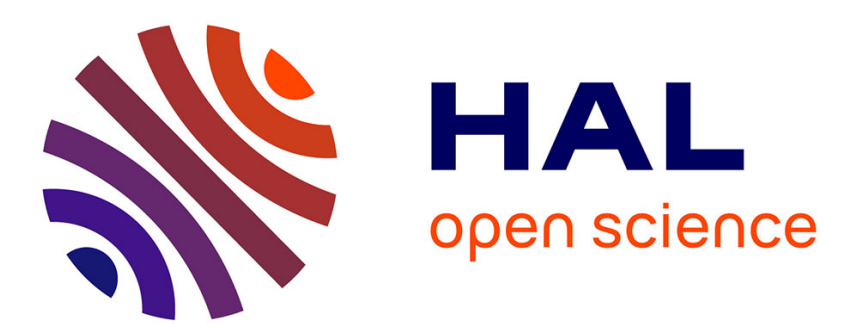

\title{
Direct and specific identification of in biological samples using fluorescently labelled DNA probes
}

\author{
M. L. Martins, A. S. Ferreira, A. Sampaio, R. Vieira, J. Inácio
}

\section{To cite this version:}

M. L. Martins, A. S. Ferreira, A. Sampaio, R. Vieira, J. Inácio. Direct and specific identification of in biological samples using fluorescently labelled DNA probes. European Journal of Clinical Microbiology and Infectious Diseases, 2010, 29 (5), pp.571-576. 10.1007/s10096-010-0897-z * hal-00575680

\author{
HAL Id: hal-00575680 \\ https://hal.science/hal-00575680
}

Submitted on 11 Mar 2011

HAL is a multi-disciplinary open access archive for the deposit and dissemination of scientific research documents, whether they are published or not. The documents may come from teaching and research institutions in France or abroad, or from public or private research centers.
L'archive ouverte pluridisciplinaire HAL, est destinée au dépôt et à la diffusion de documents scientifiques de niveau recherche, publiés ou non, émanant des établissements d'enseignement et de recherche français ou étrangers, des laboratoires publics ou privés. 


\title{
Direct and specific identification of Cryptococcus neoformans in biological samples using fluorescently labelled DNA probes
}

\author{
M. L. Martins • A. S. Ferreira • A. Sampaio • R. Vieira • \\ J. Inácio
}

Received: 30 August 2009/Accepted: 16 February 2010/Published online: 11 March 2010

(C) Springer-Verlag 2010

\begin{abstract}
Fluorescence in situ hybridisation (FISH) is a suitable technique for the rapid, reliable and cultivationindependent identification of microbial pathogens. This study describes the development of fluorescently labelled rRNA-targeted oligonucleotides and a FISH assay to detect and identify Cryptococcus neoformans in culture and biological samples. All C. neoformans reference and
\end{abstract}

M. L. Martins · A. S. Ferreira

Instituto de Higiene e Medicina Tropical,

Universidade Nova de Lisboa (UNL),

1349-008 Lisboa, Portugal

\section{L. Martins}

Centro de Recursos Microbiológicos (CREM),

Departamento de Ciências da Vida,

Faculdade de Ciências e Tecnologia,

Universidade Nova de Lisboa (UNL),

2829-516 Caparica, Portugal

A. S. Ferreira $\cdot$ A. Sampaio

Centro de Investigação e de Tecnologias Agroambientais e

Biológicas (CITAB), Univ. Trás-os-Montes e Alto Douro,

Apartado 1013,

5001-911 Vila Real, Portugal

R. Vieira

Serviço de Dermatologia, Hospital Curry Cabral,

1069-166 Lisboa, Portugal

J. Inácio

Laboratório Nacional de Investigação Veterinária (LNIV-INRB, I.P.),

Estrada de Benfica 701,

1549-011 Lisboa, Portugal

M. L. Martins $(\bowtie)$

Lab. Micologia, Instituto de Higiene e Medicina Tropical,

Universidade Nova de Lisboa (IHMT/UNL),

Rua da Junqueira, no. 96,

1349-008 Lisboa, Portugal

e-mail: luz@ihmt.unl.pt clinical isolates gave positive signals with the specific oligonucleotide probes, whereas all non-target yeast species gave negative reactions with the same probes. The assay was also successfully applied to the detection of $C$. neoformans cells in cerebrospinal samples from patients with clinical diagnosis of cryptococcosis. The described FISH-based assay revealed to be practical, sensitive and specific for the detection and identification of $C$. neoformans yeasts.

\section{Introduction}

Cryptococcus neoformans is an encapsulated basidiomycetous yeast present in the environment worldwide. Traditionally, $C$. neoformans has been classified into five serotypes, i.e. A (C. neoformans var. grubii), D ( $C$. neoformans var. neoformans), AD (hybrids of serotypes A and $\mathrm{D}$ which have antigens of the polysaccharide capsule related to those of the former), B and C (previously known as $C$. neoformans var. gattii). Currently, the members of serotypes $\mathrm{B}$ and $\mathrm{C}$ are considered to belong to a phylogenetically related but distinct species, C. gattii. The inhalation of $C$. neoformans sensu lato usually results in a self-limited asymptomatic pulmonary infection. However, C. neoformans can also cause disseminated disease, particularly in patients with depressed cell-mediated immunity [1], the meninges being the most common site of extrapulmonary infection. The diagnosis of cryptococcosis is based either on direct microscopic examination of clinical samples, such as cerebrospinal fluid (CSF), or on culture and subsequent phenotypic identification of the aetiological agent. In addition to ambiguous results, those conventional assays are time-consuming and lack sensitivity and specificity [1]. Fluorescence in situ hybridisation (FISH) has 
Table 1 List of strains and species used for the optimisation of the fluorescence in situ hybridisation (FISH) assay and respective hybridisation results

Species Strain $\quad$ Serotype $^{\mathrm{a}}$ Origin $\quad$ Hybridisation results

\begin{tabular}{|c|c|c|c|c|c|c|c|}
\hline \multirow[t]{33}{*}{$\begin{array}{l}\text { Cryptococcus } \\
\text { neoformans }\end{array}$} & $\begin{array}{l}\mathrm{CN} 137^{\mathrm{T}}(= \\
\text { PYCC } 3957^{\mathrm{T}} \text {; } \\
\left.\text { CBS } 132^{\mathrm{T}}\right)\end{array}$ & $\mathrm{D}$ & Fermenting fruit juice & + & + & + & - \\
\hline & $\mathrm{CN} 4$ & A & $\begin{array}{l}\text { CSF of an HIV 2-infected patient, Guine Bissau } \\
\text { (1992) }\end{array}$ & + & + & + & - \\
\hline & $\mathrm{CN} 23$ & A & $\begin{array}{l}\text { CSF of an HIV 1-infected patient, Curry Cabral's } \\
\text { Hospital, Lisbon (1995) }\end{array}$ & + & + & + & - \\
\hline & $\mathrm{CN} 27$ & A & CSF of an HIV 1-infected patient, Angola (1995) & + & + & + & - \\
\hline & $\mathrm{CN} 34$ & A & $\begin{array}{l}\text { CSF of an HIV 1-infected patient, Military's } \\
\text { Hospital, Lisbon (1995) }\end{array}$ & + & + & + & - \\
\hline & $\mathrm{CN} 38$ & $\mathrm{AD}$ & $\begin{array}{l}\text { Blood of an HIV 1-infected patient, Santa } \\
\text { Maria's Hospital, Lisbon (1996) }\end{array}$ & + & + & + & - \\
\hline & $\mathrm{CN} 40$ & $\mathrm{AD}$ & $\begin{array}{l}\text { CSF of an HIV 1-infected patient, Santa Maria's } \\
\text { Hospital, Lisbon (1996) }\end{array}$ & + & + & + & - \\
\hline & CN43 & A & $\begin{array}{l}\text { CSF of an HIV 1-infected patient, Institute of } \\
\text { Hygiene and Tropical Medicine, Lisbon (1996) }\end{array}$ & + & + & + & - \\
\hline & $\mathrm{CN} 48$ & D & $\begin{array}{l}\text { Skin of an HIV 1-infected patient, Curry Cabral's } \\
\text { Hospital, Lisbon (1996) }\end{array}$ & + & + & + & - \\
\hline & CN52 & $\mathrm{AD}$ & $\begin{array}{l}\text { Blood of an HIV 1-infected patient, Santa Maria's } \\
\text { Hospital, Lisbon (1996) }\end{array}$ & + & + & + & - \\
\hline & CN54 & A & $\begin{array}{l}\text { CSF of an HIV 1-infected patient, Cascais's } \\
\text { Hospital, Cascais, Portugal (1996) }\end{array}$ & + & + & + & - \\
\hline & CN55 & A & $\begin{array}{l}\text { CSF of an HIV 1-infected patient, St. António's } \\
\text { Hospital, Oporto, Portugal (1996) }\end{array}$ & + & + & + & - \\
\hline & CN57 & A & $\begin{array}{l}\text { CSF of an HIV 1-infected patient, Cascais's } \\
\text { Hospital, Cascais (1996) }\end{array}$ & + & + & + & - \\
\hline & CN59 & A & $\begin{array}{l}\text { CSF of an HIV 1-infected patient, Santa Maria's } \\
\text { Hospital, Lisbon (1996) }\end{array}$ & + & + & + & - \\
\hline & CN65 & A & $\begin{array}{l}\text { CSF of an HIV 1-infected patient, St Antonio's } \\
\text { Hospital, Oporto, Portugal (1996) }\end{array}$ & + & + & + & - \\
\hline & CN68 & $\mathrm{D}$ & $\begin{array}{l}\text { Skin of an HIV 1-infected patient, Egas Moniz's } \\
\text { Hospital, Lisbon (1996) }\end{array}$ & + & + & + & - \\
\hline & CN73 & $\mathrm{AD}$ & $\begin{array}{l}\text { Skin of an HIV 1-infected patient, Curry Cabral's } \\
\text { Hospital, Lisbon (1996) }\end{array}$ & + & + & + & - \\
\hline & CN80 & $\mathrm{D}$ & $\begin{array}{l}\text { CSF of an HIV 1-infected patient, Institute } \\
\text { Pasteur, Paris (1997) }\end{array}$ & + & + & + & - \\
\hline & CN88 & A & $\begin{array}{l}\text { CSF of an HIV-infected patient, St. António’s } \\
\text { Hospital, Oporto (1997) }\end{array}$ & + & + & + & - \\
\hline & CN95 & A & $\begin{array}{l}\text { CSF of an HIV 1-infected patient, Caxias } \\
\text { Prisoner Hospital, Lisbon (1997) }\end{array}$ & + & + & + & - \\
\hline & CN100 & A & $\begin{array}{l}\text { CSF of an HIV 1-infected patient, St. José's } \\
\text { Hospital, Lisbon (1997) }\end{array}$ & + & + & + & - \\
\hline & CN107 & $\mathrm{AD}$ & $\begin{array}{l}\text { CSF of an HIV 1-infected patient, S. Francisco } \\
\text { Xavier's Hospital, Lisbon (1997) }\end{array}$ & + & + & + & - \\
\hline & CN112 & A & Pigeon faeces, Lisbon & + & + & + & - \\
\hline & $\mathrm{CN} 312$ & nd & CSF, St. António’s Hospital, Oporto (2008) & nd & + & + & - \\
\hline & $\mathrm{CN} 313$ & nd & CSF, St. António’s Hospital, Oporto (2008) & nd & + & + & - \\
\hline & $\mathrm{CN} 314$ & nd & $\begin{array}{l}\text { Bronchial aspirate, St. António’s Hospital, } \\
\text { Oporto (2008) }\end{array}$ & nd & + & + & - \\
\hline & $\mathrm{CN} 315$ & nd & Blood, St. António’s Hospital, Oporto (2008) & nd & + & + & - \\
\hline & $\mathrm{CN} 316$ & nd & CSF, St. António’s Hospital, Oporto (2008) & nd & + & + & - \\
\hline & $\mathrm{CN} 317$ & nd & CSF, St. António’s Hospital, Oporto (2008) & nd & + & + & - \\
\hline & $\mathrm{CN} 318$ & nd & CSF, St. António’s Hospital, Oporto (2008) & nd & + & + & - \\
\hline & CN319 & nd & CSF, St. António’s Hospital, Oporto (2008) & nd & + & + & - \\
\hline & CN320 & nd & CSF, St. António’s Hospital, Oporto (2008) & nd & + & + & - \\
\hline & $\mathrm{CN} 321$ & nd & CSF, St. António’s Hospital, Oporto (2008) & nd & + & + & - \\
\hline
\end{tabular}


Table 1 (continued)

\begin{tabular}{|c|c|c|c|c|c|c|c|}
\hline \multirow[t]{2}{*}{ Species } & \multirow[t]{2}{*}{ Strain } & \multirow[t]{2}{*}{ Serotype $^{\mathrm{a}}$} & \multirow[t]{2}{*}{ Origin } & \multicolumn{4}{|c|}{ Hybridisation results } \\
\hline & & & & Cne448 & Cne205 & EUK516 & EUB338 \\
\hline & $\mathrm{CN} 322$ & nd & CSF, St. António’s Hospital, Oporto (2008) & nd & + & + & - \\
\hline & CN323 & nd & CSF, St. António's Hospital, Oporto (2008) & nd & + & + & - \\
\hline & CN324 & nd & CSF, St. António's Hospital, Oporto (2008) & nd & + & + & - \\
\hline & CN325 & nd & CSF, St. António's Hospital, Oporto (2008) & nd & + & + & - \\
\hline & CN326 & nd & CSF, St. António's Hospital, Oporto (2008) & nd & + & + & - \\
\hline & $\mathrm{CN} 327$ & nd & CSF, St. António’s Hospital, Oporto (2008) & nd & + & + & - \\
\hline & $\mathrm{CN} 328$ & nd & CSF, St. António's Hospital, Oporto (2008) & nd & + & + & - \\
\hline & CN329 & nd & CSF, St. António’s Hospital, Oporto (2008) & nd & + & + & - \\
\hline & $\mathrm{CN} 330$ & nd & CSF, St. António’s Hospital, Oporto (2008) & nd & + & + & - \\
\hline & $\mathrm{CN} 331$ & nd & CSF, St. António’s Hospital, Oporto (2008) & nd & + & + & - \\
\hline & $\mathrm{CN} 332$ & nd & CSF, St. António’s Hospital, Oporto (2008) & nd & + & + & - \\
\hline & $\mathrm{CN} 333$ & nd & CSF, St. António’s Hospital, Oporto (2008) & nd & + & + & - \\
\hline & $\mathrm{CN} 334$ & nd & CSF, St. António’s Hospital, Oporto (2008) & nd & + & + & - \\
\hline & $\mathrm{CN} 335$ & nd & Blood, St. António's Hospital, Oporto (2008) & nd & + & + & - \\
\hline & $\mathrm{CN} 336$ & nd & CSF, St. António’s Hospital, Oporto (2008) & nd & + & + & - \\
\hline & $\mathrm{CN} 337$ & nd & $\begin{array}{l}\text { Bronchoalveolar lavage, St. António's Hospital, } \\
\text { Oporto (2008) }\end{array}$ & nd & + & + & - \\
\hline & $\mathrm{CN} 338$ & nd & Urine, St. António’s Hospital, Oporto (2008) & nd & + & + & - \\
\hline & CN339 & nd & CSF, St. António’s Hospital, Oporto (2008) & nd & + & + & - \\
\hline & $\mathrm{CN} 340$ & nd & Blood, St. António’s Hospital, Oporto (2008) & nd & + & + & - \\
\hline & $\mathrm{CN} 341$ & nd & CSF, St. António’s Hospital, Oporto (2008) & nd & + & + & - \\
\hline & $\mathrm{CN} 342$ & nd & CSF, St. António’s Hospital, Oporto (2008) & nd & + & + & - \\
\hline & CN343 & nd & CSF, St. António’s Hospital, Oporto (2008) & nd & + & + & - \\
\hline & CN344 & nd & CSF, St. António’s Hospital, Oporto (2008) & nd & + & + & - \\
\hline & CN345 & nd & CSF, St. António’s Hospital, Oporto (2008) & nd & + & + & - \\
\hline & CN346 & nd & CSF, St. António’s Hospital, Oporto (2008) & nd & + & + & - \\
\hline & CN347 & nd & CSF, St. António’s Hospital, Oporto (2008) & nd & + & + & - \\
\hline & $\mathrm{CN} 348$ & nd & CSF, St. António’s Hospital, Oporto (2008) & nd & + & + & - \\
\hline & CN349 & nd & CSF, St. António’s Hospital, Oporto (2008) & nd & + & + & - \\
\hline \multirow[t]{2}{*}{$\begin{array}{l}\text { Cryptococcus } \\
\text { gattii }\end{array}$} & $\begin{array}{l}\text { CN139 } \\
(=\text { PYCC } \\
5025)\end{array}$ & B & Eucalyptus, Australia & nd & + & + & - \\
\hline & CBS 10101 & $\mathrm{C}$ & Cheata, South Africa & nd & + & + & - \\
\hline $\begin{array}{l}\text { Candida } \\
\text { albicans }\end{array}$ & $\begin{array}{l}\text { IHMT 2588/ } \\
96\end{array}$ & na & Human oesophagus biopsy (1996) & - & - & + & - \\
\hline $\begin{array}{r}\text { Candida } \\
\text { curvata }\end{array}$ & $\begin{array}{l}\text { IHMT 658/ } \\
97\end{array}$ & na & Human vaginal exudate (1997) & - & - & + & - \\
\hline $\begin{array}{l}\text { Candida } \\
\text { famata }\end{array}$ & $\begin{array}{l}\text { IHMT 415/ } \\
97\end{array}$ & na & Human faeces (1998) & - & - & + & - \\
\hline \multirow[t]{2}{*}{$\begin{array}{l}\text { Candida } \\
\text { glabrata }\end{array}$} & $\begin{array}{l}\text { PYCC } 2418^{\mathrm{T}} \\
(=\mathrm{CBS} \\
\left.138^{\mathrm{T}}\right)\end{array}$ & na & Human faeces & - & - & + & - \\
\hline & $\begin{array}{l}\text { IHMT } 128 / \\
98\end{array}$ & na & Human vaginal exudate (1998) & - & - & + & - \\
\hline $\begin{array}{l}\text { Candida } \\
\text { guilliermondii }\end{array}$ & IHMT 45/99 & na & Blood (1999) & - & - & + & - \\
\hline Candida kefyr & $\begin{array}{l}\text { IHMT } 422 / \\
98\end{array}$ & na & Human vaginal exudate (1998) & - & - & + & - \\
\hline Candida krusei & $\begin{array}{l}\text { IHMT 527/ } \\
97\end{array}$ & na & Human vaginal exudate (1997) & - & - & + & - \\
\hline $\begin{array}{l}\text { Candida } \\
\text { tropicalis }\end{array}$ & IHMT 388/98 & na & Human vaginal exudate (1998) & - & - & + & - \\
\hline
\end{tabular}


Table 1 (continued)

\begin{tabular}{|c|c|c|c|c|c|c|c|}
\hline \multirow[t]{2}{*}{ Species } & \multirow[t]{2}{*}{ Strain } & \multirow[t]{2}{*}{ Serotype $^{a}$} & \multirow[t]{2}{*}{ Origin } & \multicolumn{4}{|c|}{ Hybridisation results } \\
\hline & & & & Cne448 & Cne205 & EUK516 & EUB338 \\
\hline $\begin{array}{l}\text { Candida } \\
\text { parapsilosis }\end{array}$ & IHMT 195/98 & na & Human vaginal exudate (1998) & - & - & + & - \\
\hline \multirow[t]{2}{*}{$\begin{array}{l}\text { Cryptococcus } \\
\text { albidus }\end{array}$} & $\begin{array}{l}\text { PYCC } 2409^{\mathrm{T}} \\
\left(=\operatorname{CBS} 142^{\mathrm{T}}\right)\end{array}$ & na & Atmosphere (Japan) & - & - & + & - \\
\hline & CN71 & na & Blood & - & - & + & - \\
\hline $\begin{array}{l}\text { Cryptococcus } \\
\text { laurentii }\end{array}$ & $\begin{array}{l}\text { PYCC } 3966^{\mathrm{T}} \\
\left(=\operatorname{CBS} 139^{\mathrm{T}}\right)\end{array}$ & na & Palmwine (Congo) & - & - & + & - \\
\hline $\begin{array}{l}\text { Debaryomyces } \\
\text { hansenii }\end{array}$ & CN15 & na & Pigeon excreta & - & - & + & - \\
\hline Rhodotorula glutinis & $\begin{array}{r}\operatorname{PYCC~} 4177^{\mathrm{T}} \\
\left(=\mathrm{CBS} 20^{\mathrm{T}}\right)\end{array}$ & na & Atmosphere & - & - & + & - \\
\hline $\begin{array}{l}\text { Saccharomyces } \\
\text { cerevisiae }\end{array}$ & $\begin{array}{l}\text { PYCC } 4455^{\mathrm{T}} \\
\left(=\operatorname{CBS} 1171^{\mathrm{T}}\right)\end{array}$ & na & Brewer's top yeast (Netherlands) & - & - & + & - \\
\hline $\begin{array}{l}\text { Sirobasidium } \\
\text { magnum }\end{array}$ & PYCC 5289 & na & Unknown & - & - & + & - \\
\hline $\begin{array}{l}\text { Trichosporon } \\
\text { cutaneum }\end{array}$ & IHMT 256/97 & na & Human vaginal exudate (1997) & - & - & + & - \\
\hline
\end{tabular}

${ }^{\text {a }}$ According to Martins [6]

nd = not determined; na $=$ not applicable; $\mathrm{T}=$ type strain; $\mathrm{CSF}=$ cerebrospinal fluid

All strains are maintained at the Mycology Lab ('CN' and 'IHMT' strains) of the Institute of Hygiene and Tropical Medicine (IHMT/UNL), Lisbon, Portugal, at the Portuguese Yeast Culture Collection ('PYCC' strains), Caparica, Portugal, and at the Centraalbureau voor Schimmelcultures ('CBS' strains), Utrecht, The Netherlands

been widely used for the detection and identification of microorganisms, with emphasis on prokaryotic organisms, allowing the direct microscopic visualisation and quantification of individual cells in their natural environments [2,3]. FISH has also been used to detect and identify fungal organisms, including clinically relevant yeasts such as Candida species $[4,5]$. The aim of this study was to optimise and evaluate the practicability, sensitivity and specificity of a FISH-based assay for the identification of $C$. neoformans directly in clinical samples.

\section{Materials and methods}

Sixty-one C. neoformans and two C. gattii isolates of several origins, most of them obtained from human immunodeficiency virus (HIV)-positive patients, were used in this work (Table 1). Eighteen strains belonging to other yeasts species, including clinical isolates, were used as negative controls (Table 1). All yeasts were grown aerobically under continuous shaking in $\mathrm{YM}$ broth $(0.3 \% \mathrm{w} / \mathrm{v}$ malt extract, $0.2 \%$ yeast extract, $0.5 \%$ peptone, $1 \%$ glucose) at $25^{\circ} \mathrm{C}$. Cells were harvested in the exponential growth phase (optical density of 2.5 at $600 \mathrm{~nm}$ ) by centrifugation for $5 \mathrm{~min}$ at $13,000 \mathrm{rpm}$. Cells were washed once with $1 \times$ phosphate-buffered saline (PBS) $(130 \mathrm{mM}$ sodium chloride, $10 \mathrm{mM}$ sodium phosphate buffer, $\mathrm{pH}$ 7.2) and fixed for $4 \mathrm{~h}$ with $4 \% \mathrm{w} / \mathrm{v}$ paraformaldehyde in PBS at $4^{\circ} \mathrm{C}$. After fixation, cells were washed twice with PBS, resuspended in one volume of PBS and one volume of cold absolute ethanol and stored at $-20^{\circ} \mathrm{C}$ until use. A total of $18 \mathrm{CSF}$ and 10 blood samples from HIVpositive patients with clinical diagnosis of cryptococcosis received for routine conventional testing at the Institute of Hygiene and Tropical Medicine and at the Hospital Santa Maria, both in Lisbon, were also included in this study. Additionally, negative CSF samples were artificially infected with C. neoformans PYCC $3957^{\mathrm{T}}$ type strain (seven samples) or other clinically relevant yeasts species (seven samples) and used as positive and negative controls, respectively. Each biological sample was also examined for the presence of encapsulated budding yeast by India ink preparation using microscopy (CSF samples), for the presence of cryptococcal polysaccharide antigen by latex agglutination test (Pastorex Crypto Plus, Bio-Rad) (CSF and blood samples) and by culture on Sabouraud glucose agar (CSF samples), according to standard procedures $[1,6]$. Additionally, $1 \mathrm{ml}$ of each CSF sample was centrifuged at $13,000 \mathrm{rpm}$ for $5 \mathrm{~min}$ to pellet yeast cells, which was then washed once with $500 \mu \mathrm{l}$ of $1 \times$ PBS and fixed with $4 \%$ paraformaldehyde as described above.

Blood samples were treated as previously described [7] and the resulting lysates were treated as for CSF samples. Two 26S rRNA-targeted species-specific oligonucleotide probes were designed for $C$. neoformans, which are also complementary to the closely related $C$. gattii: Cne448 (5'CCAGCCCTTATCCACCGA) and Cne205 (5'- 
GTCGCGTTACTTGGGAGT). Probes EUK516 (5'-ACCA GACTTGCCCTCC), universal for eukaryotic organisms, and EUB338 (5'-GCTGCCTCCCGTAGGAGT), targeting all bacteria, were used as positive and negative hybridisation controls, respectively [8]. All probes were labelled at their 5'end with the fluorochrome Cy3 (MWG Biotech, Ebersberg, Germany). Approximately $10^{6}$ fixed yeast cells obtained from cultures, and whole fixed pellets obtained from clinical samples, were hybridised in $20 \mu \mathrm{l}$ of hybridisation buffer $(0.9 \mathrm{M} \mathrm{NaCl}, 0.01 \% \mathrm{w} / \mathrm{v}$ SDS, $20 \mathrm{mM}$ Tris-HCl, $\mathrm{pH} 7.2$ and $45 \%$ formamide for Cne $448,10 \%$ for Cne 205 and 20\% for EUK516 and EUB338), with $1.5 \mathrm{ng}$ of Cy3-labelled probe and incubated at $46^{\circ} \mathrm{C}$ for $2 \mathrm{~h}$. After incubation, cells were pelleted by centrifugation and resuspended in $1 \mathrm{ml}$ of prewarmed washing buffer $(20 \mathrm{mM}$ Tris- $\mathrm{HCl}, \mathrm{pH}$ 8.0, $0.01 \% \mathrm{w} / \mathrm{v}$ SDS, $5 \mathrm{mM}$ EDTA and $400 \mathrm{mM} \mathrm{NaCl}$ for Cne448, $125 \mathrm{mM}$ for Cne205 and $225 \mathrm{mM}$ for EUK516 and EUB338 probes) for $30 \mathrm{~min}$ at $46^{\circ} \mathrm{C}$. The suspension was then centrifuged and the pellet resuspended in $50 \mu \mathrm{l}$ of $1 \times$ PBS, placed on ice and analysed within $3 \mathrm{~h}$. For each sample, $10 \mu \mathrm{l}$ of hybridised suspension was spotted onto precleaned microscopic slides and dried at $37^{\circ} \mathrm{C}$ for $20 \mathrm{~min}$. The slides were then mounted with Vectashield solution (Vector, Burlingame, CA, USA) and examined with an Olympus BX50 microscope, fitted for epifluorescence microscopy with a U-ULH $100 \mathrm{~W}$ mercury high-pressure bulb and a U-MA1007 filter set for the fluorochrome Cy3 (Olympus). Microphotographs were taken using a digital camera (Olympus C3030-Z00M) and edited using standard software (Adobe Photoshop 6.0, Adobe).

\section{Results and discussion}

Positive hybridisation signals were determined as bright orange fluorescent yeast cells. All 61 C. neoformans and
Table 2 List of cerebrospinal fluid (CSF) and blood samples analysed by conventional diagnostic methods and by FISH with the probe Cne205
${ }^{\text {a }}$ Positive: detection of encapsulated yeast cells on samples

${ }^{\mathrm{b}}$ Positive: Cryptococcus neoformans colonies detected

${ }^{\mathrm{c}}$ Positive: detection of $C$. neoformans antigen presence in the clinical sample

${ }^{\mathrm{d}}$ Detection of fluorescent yeast cells with FISH probe Cne205: +

+ , bright and intense fluorescently labelled yeast cells; + , yeast cells with a faint fluorescent signal;

Negative, no fluorescent yeast cells detected

$\mathrm{CSF}=$ cerebrospinalfluid; nd $=$ not determined

\begin{tabular}{|c|c|c|c|c|c|}
\hline IHMT no. & Sample & $\begin{array}{l}\text { Microscopy } \\
\text { with India ink }\end{array}$ & $\begin{array}{l}\text { Sabouraud } \\
\text { culture }^{b}\end{array}$ & $\begin{array}{l}\text { Title of cryptococcal } \\
\text { polysaccharide } \\
\text { antigen }^{c}\end{array}$ & $\mathrm{FISH}^{\mathrm{d}}$ \\
\hline $383 / 97$ & CSF & Negative & Negative & $1: 10,000$ & ++ \\
\hline $393 / 97$ & $\mathrm{CSF}$ & Negative & Negative & $1: 1,000$ & ++ \\
\hline $397 / 97$ & $\mathrm{CSF}$ & Negative & Positive & $1: 10,000$ & ++ \\
\hline $421 / 97$ & $\mathrm{CSF}$ & Positive & Negative & $1: 10,000$ & ++ \\
\hline $580 / 97$ & CSF & Positive & Positive & $1: 1,000$ & ++ \\
\hline $594 / 97$ & $\mathrm{CSF}$ & Positive & Negative & $1: 10,000$ & ++ \\
\hline $600 / 97$ & $\mathrm{CSF}$ & Positive & Positive & $1: 10,000$ & ++ \\
\hline $37 / 99$ & CSF & Positive & Positive & $1: 10,000$ & ++ \\
\hline $59 / 99$ & CSF & Negative & Negative & Negative & Negative \\
\hline 277/99 & CSF & Positive & Positive & $1: 10,000$ & ++ \\
\hline 99/01 & CSF & Positive & Positive & $1: 2,048$ & ++ \\
\hline $167 / 01$ & CSF & Positive & Positive & $1: 1,000$ & ++ \\
\hline $186 / 01$ & $\mathrm{CSF}$ & Positive & Positive & $1: 10,000$ & ++ \\
\hline $190 / 01$ & $\mathrm{CSF}$ & Negative & Negative & Negative & Negative \\
\hline 197/01 & CSF & Negative & Negative & Negative & Negative \\
\hline $202 / 01$ & $\mathrm{CSF}$ & Negative & Negative & Negative & Negative \\
\hline $54 / 02$ & CSF & Positive & Positive & $1: 1,000$ & ++ \\
\hline $274 / 02$ & CSF & Negative & Positive & $1: 1,500$ & ++ \\
\hline $394 / 97$ & Blood & nd & nd & $1: 10,000$ & + \\
\hline $523 / 97$ & Blood & nd & nd & $1: 10,000$ & + \\
\hline $525 / 97$ & Blood & nd & nd & $1: 10$ & Negative \\
\hline $595 / 97$ & Blood & nd & nd & $1: 10,000$ & + \\
\hline $597 / 97$ & Blood & nd & nd & $1: 10,000$ & + \\
\hline $621 / 97$ & Blood & nd & nd & $1: 1,000$ & + \\
\hline $625 / 97$ & Blood & nd & nd & $1: 1,000$ & + \\
\hline $187 / 01$ & Blood & nd & nd & $1: 10,000$ & + \\
\hline $110 / 02$ & Blood & nd & nd & $1: 100$ & + \\
\hline $275 / 02$ & Blood & nd & nd & $1: 1,000$ & Negative \\
\hline
\end{tabular}


two C. gattii isolates gave positive signals with Cne 448 and/or Cne205 probes, whereas all non-target species gave negative reactions with the same probes (Table 1). It was also possible to identify $C$. neoformans cells with the specific probes in mixed samples with other yeast species. All yeast species tested yielded strong fluorescent signals with the universal probe EUK516. None of the yeast cells showed any fluorescent signal with the EUB338 probe (Table 1). FISH with the Cne205 probe always showed stronger fluorescent signals than with the Cne448 probe. This can be explained since the complementary target of the later probe is located in a low-accessibility rRNA region for FISH [9]. Probe Cne205, which presented the brightest fluorescent signals, was evaluated for the detection of $C$. neoformans in clinical samples. A group of cerebrospinal and serum fluid samples was firstly examined and the direct identification of $C$. neoformans cells in all artificially infected samples was achieved. The assay was then applied in cerebrospinal and serum samples from patients with clinical diagnosis of cryptococcosis, in parallel with other conventional diagnostic methods (Table 2). Ten culturepositive CSF samples revealed to be also positive by FISH. Additionally, four culture-negative CSF samples revealed to be FISH-positive (two of these samples were also positive by direct microscopy with India ink; cryptococcal antigens were detected in all four). The detection limit of our Cne205 probe-based FISH assay was estimated to be $10^{2}$ cells $/ \mathrm{ml}$ when using serially diluted artificially spiked CSF samples (data not shown). Four CSF samples were negative by all tests performed. The detection of $C$. neoformans from blood samples was more difficult, with cells presenting less intense fluorescence signals (Table 2).

Other groups have recently reported positive outcomes on the introduction of FISH-based assays in the routine of their clinical laboratories $[4,10]$. The incorporation of these assays as part of the initial yeast laboratorial identification algorithm can result in substantial savings for hospitals [4]. FISH associates the fastness, sensitivity and specificity of molecular diagnostic methods with the observation of morphologic features of the aetiological agents of disease, contributing to a reliable identification of the pathogens directly in their substrates and leading to the consequent definition of the most appropriate treatment. To the best of our knowledge, we describe in this study the first FISHbased assay for the detection and identification of $C$. neoformans yeasts, which revealed to be practical, sensitive and specific when compared to the conventional methods. FISH may constitute a valuable complementary tool to conventional $C$. neoformans laboratory analysis, especially when using CSF samples. It can be automated and implemented in hospital laboratories with a great number of analyses per day.

Acknowledgements We thank Maria José Salgado and Lurdes Monteiro (Hospital de Santa Maria, Lisbon), Zélia Saraiva and Teresa Tendeiro (Instituto Português de Oncologia, Lisbon), and Virgínia Lopes (Hospital de Santo António, Oporto) for providing the yeast isolates and/or clinical samples. The authors would also like to acknowledge Prof. Isabel Spencer-Martins, prematurely deceased on June 15 th 2008 , for her valuable suggestions on the present work.

\section{References}

1. Casadevall A, Perfect JR (1998) Cryptococcus neoformans. ASM Press, Washington

2. Amann R, Fuchs BM (2008) Single-cell identification in microbial communities by improved fluorescence in situ hybridization techniques. Nat Rev Microbiol 6:339-348

3. Amann R, Ludwig W, Schleifer K-H (1995) Phylogenetic identification and in situ detection of individual microbial cells without cultivation. Microbiol Rev 59:143-169

4. Alexander BD, Ashley ED, Reller LB, Reed SD (2006) Cost savings with implementation of PNA FISH testing for identification of Candida albicans in blood cultures. Diagn Microbiol Infect Dis 54:277-282

5. Gherna M, Merz WG (2009) Identification of Candida albicans and Candida glabrata within 1.5 hours directly from positive blood culture bottles with a shortened peptide nucleic acid fluorescence in situ hybridization protocol. J Clin Microbiol 47:247-248

6. Martins ML (2001) Polimorfismos fenéticos e diagnóstico molecular de Cryptococcus neoformans. PhD Dissertation, Universidade Nova de Lisboa (in Portuguese)

7. Lischewski A, Kretschmar M, Hof H, Amann R, Hacker J, Morschhäuser J (1997) Detection and identification of Candida species in experimentally infected tissue and human blood by rRNA-specific fluorescent in situ hybridization. J Clin Microbiol 35:2943-2948

8. Amann RI, Binder BJ, Olson RJ, Chisholm SW, Devereux R, Stahl DA (1990) Combination of 16S rRNA-targeted oligonucleotide probes with flow cytometry for analyzing mixed microbial populations. Appl Environ Microbiol 56:1919-1925

9. Inácio J, Behrens S, Fuchs BM, Fonseca A, Spencer-Martins I, Amann R (2003) In situ accessibility of Saccharomyces cerevisiae 26S rRNA to Cy3-labeled oligonucleotide probes comprising the D1 and D2 domains. Appl Environ Microbiol 69:2899-2905

10. Peters RP, Savelkoul PH, Simoons-Smit AM, Danner SA, Vandenbroucke-Grauls CM, van Agtmael MA (2006) Faster identification of pathogens in positive blood cultures by fluorescence in situ hybridization in routine practice. J Clin Microbiol 44:119-123 